\title{
Clinicopathological characteristics and prognosis of lung cancer in young patients aged 30 years and younger
}

\author{
Fang Li", Huayu He”, Bin Qiu, Ying Ji, Kelin Sun, Qi Xue, Wei Guo, Dali Wang, Jun Zhao, \\ Yousheng Mao, Juwei Mu, Shugeng Gao \\ Department of Thoracic Surgery, National Cancer Center/National Clinical Research Center for Cancer/Cancer Hospital, Chinese Academy of \\ Medical Sciences and Peking Union Medical College, Beijing 100021, China \\ Contributions: (I) Conception and design: F Li, H He; (II) Administrative support: S Gao; (III) Provision of study materials or patients: F Li, H He; (IV) \\ Collection and assembly of data: F Li, H He, B Qiu, Y Ji; (V) Data analysis and interpretation: F Li, H He; (VI) Manuscript writing: All authors; (VII) \\ Final approval of manuscript: All authors. \\ \#These authors contributed equally to this work. \\ Correspondence to: Shugeng Gao. Department of Thoracic Surgery, National Cancer Center/National Clinical Research Center for Cancer/Cancer \\ Hospital, Chinese Academy of Medical Sciences and Peking Union Medical College, Beijing 100021, China. Email: gaoshugeng@vip.sina.com.
}

\begin{abstract}
Background: Lung cancer is one of the most common malignant tumors in the world and mainly occurs in elderly patients, but rarely in young patients. The purpose of this retrospective study was to examine the clinicopathological features and prognosis of lung cancer patients aged 30 years and younger.

Methods: Patients aged 30 years and younger with lung cancer admitted to our center from November 2013 to October 2018 were retrospectively identified. Data included sex, age, smoking history, family history of cancer, high resolution computed tomography results, size and location of tumors, histology of tumors, lymph node status, stage of tumors, treatment methods and prognosis of patients.

Results: The patient group included more females (56.3\%) than males (43.7\%) among lung cancer patients aged 30 and younger. Some patients had a history of tobacco inhalation and family cancer $(17.5 \%$ and $22.3 \%$, respectively). The most common tumors were in the left lower lobe (27.2\%). Nearly half (49.5\%) of the patients had pathological adenocarcinomas and $59.3 \%$ of the patients were showed early clinical stage and had no lymph node metastasis. All patients received surgical treatment; $47.1 \%$ received lobectomy and only $17.9 \%$ received adjuvant therapy such as radiotherapy, chemotherapy or targeted therapy after operation. Only seven (7.4\%) of the successful follow-up patients died. Local recurrence occurred in two cases and distant metastasis in six cases.

Conclusions: The main clinicopathological type of lung cancer in young lung cancer patients aged 30 years and younger is adenocarcinoma, and most cases were at the early stage. Surgical treatment based on lobectomy is still the main treatment method and the prognosis of these patients is very good. Early screening of lung cancer should be actively promoted for young people.
\end{abstract}

Keywords: Lung cancer; young patient; clinicopathological characteristics

Submitted Aug 11, 2019. Accepted for publication Sep 16, 2019.

doi: $10.21037 /$ jtd.2019.09.60

View this article at: http://dx.doi.org/10.21037/jtd.2019.09.60

\section{Introduction}

Lung cancer is currently one of the most common malignant tumors in the world and has attracted increasing attention because of its high incidence and mortality. An estimated 2.1 million new cases of lung cancer $(11.6 \%$ of all new tumors) and 1.8 million deaths $(18.4 \%)$ were predicted for 2018 (1). Lung cancer is commonly diagnosed in elderly patients aged 70 years or older, while the incidence of lung cancer in young patients under 40 years old is relatively 


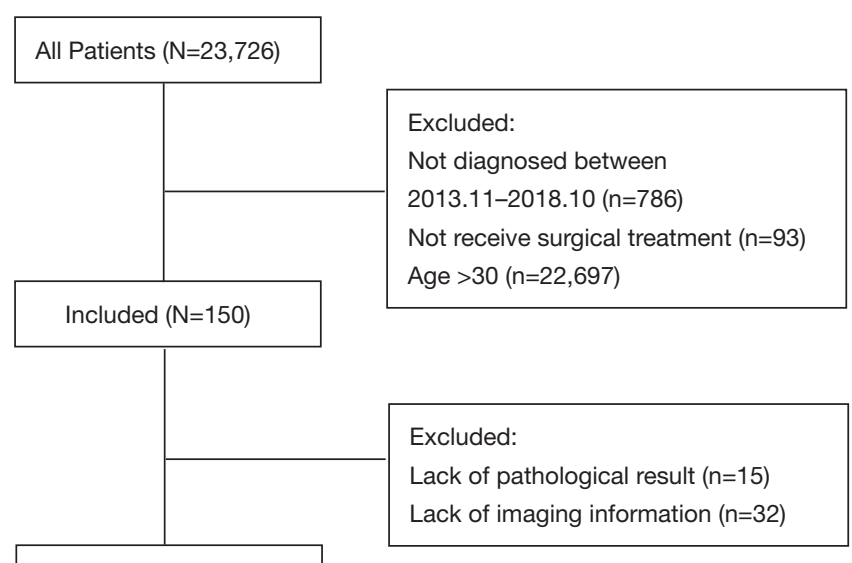

Final sample $(\mathrm{N}=103)$

Figure 1 Flowchart of exclusion criteria and study design.

low $(1-10 \%)(2,3)$. Recent studies have reported that the incidence of lung cancer in young people is increasing (4-6). Earlier studies have shown that lung cancer patients under 40 years have a higher malignancy and mortality rate than elderly patients $(7,8)$. In cases of advanced lung cancer, the prognosis is also poor, perhaps because young people have better compensatory ability for their physical functions and clinical symptoms are not obvious (9-11). Sugio et al. found that the prognosis of young patients was not worse than that of lung cancer patients over 40 years old (12) and the survival rate after treatment was higher than that of elderly patients $(13,14)$. Brian et al. found that the median survival time of lung cancer patients under 46 years old in stages I-IV was 60, 59.10, 18.4 and 8.8 months, respectively (5). Few specialized studies on younger groups of lung cancer patients are currently available. Young people under 30 years old (including 30 years old) represent upcoming generations and potential future contributors to society. Therefore, studying the characteristics and prognosis of lung cancer patients in the younger population may have significant implications. We thus conducted a retrospective study with a focus on describing and analyzing the clinicopathological features and prognosis of lung cancer patients aged 30 and younger. This is the first study specifically aimed at young lung cancer patients aged 30 and younger in China. We hope to provide constructive suggestions for the prevention and treatment of young lung cancer patients in the future by studying the clinicopathological characteristics and prognostic factors of this special group.

\section{Methods}

\section{Study population and data collection}

This study retrospectively analyzed lung cancer patients 30 years old and younger who were admitted to the Cancer Hospital of Chinese Academy of Medical Sciences, National Cancer Center of China from November 2013 to October 2018. After excluding patients whose pathological results or imaging results were non-retrievable, 103 patients were finally included in this study (Figure 1).

The clinicopathological features of the lung cancer patients, including sex, age at diagnosis, smoking history, family history of cancer, high resolution computed tomography results, tumor size and location, histology, lymph node status, tumor stage, and expressions of endothelial growth factor receptor (EGFR) and anaplastic lymphoma kinase (ALK), were reviewed and recorded. Tumor staging was mainly based on the classification criteria of tumors, lymph nodes and metastases (TNM) in the 7 th and 8 th editions of lung cancer. The immunohistochemical expression of EGFR and ALK was determined using the CDx sequence of VENTANA ALK (D5F3). Tumor histological results were strictly classified according to the 4th edition of the World Health Organization (WHO) Classification of Tumors of the Lung, Pleura, Thymus and Heart.

Of the 103 patients included in the study, 95 were followed up successfully, mainly by consulting the outpatient consultation records or contacting patients or family members by telephone and e-mail. This study was approved by the ethics committee of our institute.

\section{Statistical analysis}

All data in this study were analyzed by SPSS 23.0 (IBM Corp., Armonk, NY, USA) software. Statistical significance was set as $\mathrm{P}<0.05$. Patients were followed up until May 2019 or their death date. Total survival was defined as the time from the diagnosis date to the last follow-up or death. Progressive-free survival was defined as the time from surgery to recurrence or the last follow-up. Survival curves were plotted using the Kaplan-Meier method. Multivariate analyses for OS were performed using the Cox regression model. Factors that showed statistically significant association with survival in univariate analysis were included into multivariate Cox proportional hazards model. 


\section{Results}

\section{Epidemiological characteristics}

From November 2013 to October 2018, a total of 103 lung cancer patients under 30 years old were diagnosed and treated by the National Cancer Center/Cancer Hospital of the Chinese Academy of Medical Sciences. Patient characteristics are presented in Table 1. Patient age ranged from 11 to 30 years (mean age 26, standard deviation 2.9). The patient group included 45 males $(43.7 \%)$ and 58 females $(56.3 \%)$, and the ratio of males to females was 1:1.29. Among the 103 patients, 18 patients (17.5\%) had a history of tobacco inhalation, including 17 males (94.4\%) and one female (5.6\%). Twenty-three patients (22.3\%) had a family history of tumor.

\section{Clinicopathological features}

The tumors of 55 patients (53.4\%) were located at the right lung and those of 44 patients $(42.7 \%)$ were in the left lung. The lesions of 22 cases (21.4\%) were located at the right upper lobe, while lesions in four cases $(3.9 \%)$ were in the right middle lobe, lesions in 22 cases $(21.4 \%)$ were in the right lower lobe, lesions in 14 cases $(13.6 \%)$ were in the left upper lobe and lesions in 28 cases $(27.2 \%)$ were in the left lower lobe. Two cases (1.9\%) had lesion in the upper and lower lobes of the right lung, and five cases (4.9\%) had lesion in the middle and lower lobes of the right lung. Two cases $(1.9 \%)$ had lesion in the upper and lower lobes of left lung and four cases (3.9\%) had tumors in the main bronchus and trachea.

The tumors were divided into seven grades according to the maximum diameter of the tumors. Among them, 60 cases $(58.3 \%)$ had a maximum diameter of $0-2 \mathrm{~cm}, 17$ cases $(16.5 \%)$ had a maximum diameter of $2-3 \mathrm{~cm}, 20$ cases $(19.4 \%)$ had a maximum diameter of $3-5 \mathrm{~cm}$, four cases (3.9\%) had a maximum diameter of $5-7 \mathrm{~cm}$, and two cases $(1.9 \%)$ had a maximum diameter of more than $7 \mathrm{~cm}$.

According to the 4th WHO classification criteria for lung, pleura, thymus and cardiac tumors, 51 (49.5\%) cases were diagnosed as adenocarcinomas, including 7 (13.7\%) in situ adenocarcinomas and eight (15.7\%) minimally invasive adenocarcinomas. One case $(1.0 \%)$ was small cell carcinoma, one case $(1.0 \%)$ was large cell carcinoma, two cases $(1.9 \%)$ were adenoid cystic carcinoma, 11 cases $(10.7 \%)$ were mucoepidermoid carcinoma, eight cases (7.8\%) were carcinoid, one case $(1.0 \%)$ was sclerosing pneumocytoma, three cases $(2.9 \%)$ were diagnosed as carcinosarcoma, one case $(1.0 \%)$ was primary bronchial alveolar cell carcinoma, two cases $(1.9 \%)$ were diagnosed as atypical adenomatoid hyperplasia, two cases $(1.9 \%)$ were diagnosed as epithelial myoepithelial carcinoma, four cases $(3.9 \%)$ were inflammatory myofibroblastic tumor, and 16 cases $(15.5 \%)$ were diagnosed as metastasis.

Only 13 patients $(25.5 \%)$ of 51 patients with lung adenocarcinoma had EGFR and ALK immunohistochemical results. Among them, 10 patients $(19.6 \%)$ had positive EGFR immunohistochemical results and three patients (5.9\%) were negative for EGFR. Three cases (5.9\%) were positive for ALK immunohistochemistry and 10 cases (19.6\%) were negative.

According to TNM staging criteria, there were seven patients $(6.8 \%)$ with stage 0 , while 36 patients $(35.0 \%)$ had stage IA, 13 patients $(12.6 \%)$ had stage IB, five patients (4.9\%) had stage IIA, six patients (5.8\%) had stage II B, and 10 patients $(9.7 \%)$ had stage IIIA. No patients had stage IIIB and IV. Among the patient group, 26 cases failed to stage, including two cases (1.9\%) of atypical adenomatous hyperplasia, three cases $(3.9 \%)$ of non-typical adenomatous hyperplasia, one case of intratracheal carcinoid, four cases $(3.9 \%)$ of interstitial tumors, and 16 cases $(15.5 \%)$ of metastatic tumors.

\section{Treatment and prognostic survival}

The treatments for the young lung cancer patients are presented in Table 2. All patients (100.0\%) received surgical treatment. In addition, 36 patients $(35.0 \%)$ underwent open thoracic surgery and 67 patients $(65.0 \%)$ underwent videoassisted thoracoscopic surgery (VATS). Nearly half of the patients $(49,47.6 \%)$ underwent lobectomy (44 cases of single lobe and four cases of compound lobe), while 11 cases (10.7\%) underwent segmental resection, 28 cases $(27.2 \%)$ underwent wedge resection, nine cases $(8.7 \%)$ underwent bronchial sleeve lobectomy, three cases $(2.9 \%)$ underwent total pneumonectomy, and three cases $(2.9 \%)$ underwent standard tracheal resection. Of the patients undergoing lobectomy, 44 underwent unilobectomy and five underwent combined lobectomy. In addition, two patients underwent bilateral surgical treatment. One patient underwent wedge resection in stages because of bilateral lung metastasis of giant cell tumor of bone. The other case underwent sublobectomy combined with wedge resection because of bilateral lung metastasis of left temporal chondrosarcoma.

Of the 95 patients who were followed up successfully, the median follow-up time was 26 months (range, 
Table 1 Clinicopathologic characteristics of subjects in this study

\begin{tabular}{|c|c|}
\hline Variables & $\mathrm{N}(\%)$ \\
\hline Number & 103 \\
\hline \multicolumn{2}{|l|}{ Age, y } \\
\hline Median & 26 \\
\hline Mean \pm SD & $26 \pm 2.9$ \\
\hline Range & $11-30$ \\
\hline \multicolumn{2}{|l|}{ Sex } \\
\hline Male & $45(43.7)$ \\
\hline Female & $58(56.3)$ \\
\hline \multicolumn{2}{|l|}{ Smoking history } \\
\hline Smoker & $18(17.5)$ \\
\hline Male & $17(94.4)$ \\
\hline Female & $1(5.6)$ \\
\hline Current/former & $85(82.5)$ \\
\hline \multicolumn{2}{|l|}{ Family history of tumor } \\
\hline Yes & $23(22.3)$ \\
\hline No & $80(77.7)$ \\
\hline \multicolumn{2}{|l|}{ Tumor location } \\
\hline RUL & $22(21.4)$ \\
\hline $\mathrm{RML}$ & $4(3.9)$ \\
\hline RLL & $22(21.4)$ \\
\hline LUL & $14(13.6)$ \\
\hline LLL & $28(27.2)$ \\
\hline$R U L+R L L$ & $2(1.9)$ \\
\hline$R M L+R L L$ & $5(4.9)$ \\
\hline LUL + LLL & $2(1.9)$ \\
\hline Main bronchus/trachea & $4(3.9)$ \\
\hline \multicolumn{2}{|l|}{ Laterality } \\
\hline Left & $44(42.7)$ \\
\hline Right & $55(53.4)$ \\
\hline Other & $4(3.9)$ \\
\hline \multicolumn{2}{|l|}{ Tumor size (cm) } \\
\hline $0-2$ & $60(58.3)$ \\
\hline $2-3$ & $17(16.5)$ \\
\hline $3-5$ & $20(19.4)$ \\
\hline $5-7$ & $4(3.9)$ \\
\hline$>7$ & $2(1.9)$ \\
\hline
\end{tabular}

Table 1 (continued)
Table 1 (continued)

\begin{tabular}{|c|c|}
\hline Variables & N (\%) \\
\hline \multicolumn{2}{|c|}{ Histological type } \\
\hline$A C$ & $51(49.5)$ \\
\hline SCLC & $1(1.0)$ \\
\hline LCC & $1(1.0)$ \\
\hline ACC & $2(1.9)$ \\
\hline MC & $11(10.7)$ \\
\hline CTs & $8(7.8)$ \\
\hline SP & $1(1.0)$ \\
\hline CS & $3(2.9)$ \\
\hline PBACC & $1(1.0)$ \\
\hline AAH & $2(1.9)$ \\
\hline EMC & $2(1.9)$ \\
\hline IMT & $4(3.9)$ \\
\hline MTs & $16(15.5)$ \\
\hline \multicolumn{2}{|l|}{ EGFR in AC } \\
\hline Positive & $10(19.6)$ \\
\hline Negative & $3(5.9)$ \\
\hline Unknown & $38(74.5)$ \\
\hline \multicolumn{2}{|l|}{ ALK in AC } \\
\hline Positive & $3(5.9)$ \\
\hline Negative & $10(19.6)$ \\
\hline Unknown & $38(74.5)$ \\
\hline \multicolumn{2}{|c|}{ Clinical stage } \\
\hline 0 & $7(6.8)$ \\
\hline IA & $36(35.0)$ \\
\hline IB & $13(12.6)$ \\
\hline IIA & $5(4.9)$ \\
\hline IIB & $6(5.8)$ \\
\hline IIIA & $10(9.7)$ \\
\hline Unknown & $26(25.2)$ \\
\hline
\end{tabular}

$\mathrm{SD}$, standard deviation; RUL, right upper lobe; RML, right middle lobe; RLL, right lower lobe; LUL, left upper lobe; LLL, left lower lobe; AC, adenocarcinoma; SCLC, small cell lung cancer; LCC, large cell carcinoma; ACC, adenoid cystic carcinoma; MC, mucoepidermoid carcinoma; CTs, carcinoid tumors; SP, sclerosing pneumocytoma; CS, carcinosarcoma; PBACC, primary bronchial acinar cell carcinoma; $\mathrm{AAH}$, atypical adenomatous hyperplasia; EMC, epithelial-myoepithelial carcinoma; IMT, inflammatory myofibroblastic tumor; MTs, metastatic tumors; EGFR, endothelial growth factor receptor; ALK, anaplastic lymphoma kinase. 
Table 2 The treatment for young patients with lung cancer

\begin{tabular}{lc}
\hline Variables & $\mathrm{N}(\%)$ \\
\hline Surgical approach & $36(35.0)$ \\
OTS & $67(65.0)$ \\
VATS & \\
Surgical treatment & $28(27.2)$ \\
Wedge resection & $11(10.7)$ \\
Segmentectomy & $49(47.6)$ \\
Lobectomy & $9(8.7)$ \\
Bronchial sleeve lobe resection & $3(2.9)$ \\
Pneumonectomy & $3(2.9)$ \\
Standard tracheal resection & \\
Treatment & $75(72.8)$ \\
Surgery & $15(14.6)$ \\
Surgery + chemotherapy & $1(1.0)$ \\
Surgery + radiotherapy & $2(1.9)$ \\
Surgery + CR + MTT & $2(1.9)$ \\
Surgery + MTT & $8(7.8)$ \\
Lost to follow-up after surgery & \\
\hline & \\
\hline TS, open thoracic surgery; VATS,
\end{tabular}

OTS, open thoracic surgery; VATS, video-assisted thoracic surgery; CR, chemoradiotherapy; MTT, molecular targeted therapy.

5-65 months), 7 (7.4\%) patients died and 88 (92.6\%) patients still alive. The 1-year and 3-year survival rates for the 95 cases were $95.6 \%$ and $85.9 \%$, respectively (Figure 2). Tumor recurrence occurred in eight (8.1\%) patients, including two $(2.3 \%)$ patients who had local recurrence and six $(6.8 \%)$ patients who had distant metastasis. Two patients with local recurrence also showed distant metastasis. Of the seven patients who died, four had metastatic lung tumors at first diagnosis and two had primary lung cancer at first diagnosis but died of other tumors. Only two patients died of lung cancer. One survived for 9 months and the other survived for 32 months (Table 3). In univariate analyses, only the treatment modalities (HR =7.37, 95\% CI: 2.49-14.62, P=0.001) significantly associated with OS. Multivariate Cox regression analyses of OS found that also only treatment modalities (HR $=14.32$, $95 \% \mathrm{CI}: 1.71-120.30, \mathrm{P}=0.014$ ) was associated with survival (Table 4).

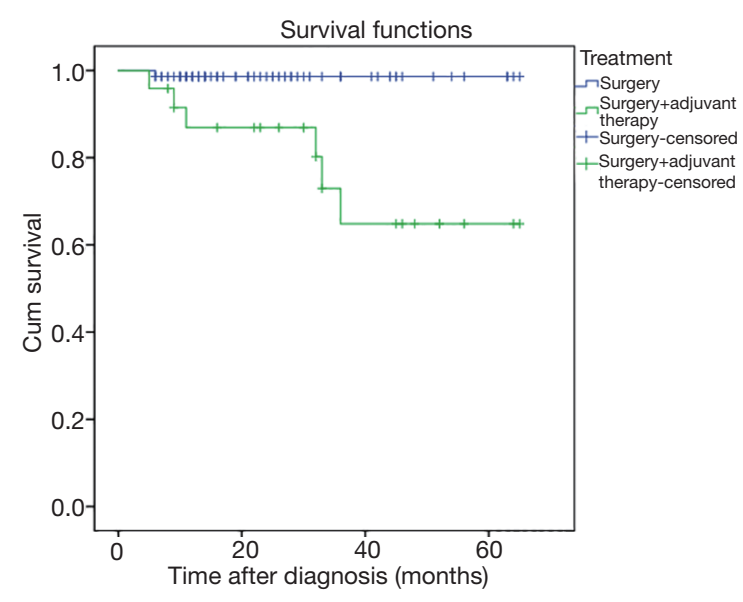

Figure 2 Kaplan-Meier curve depicting overall survival according to treatment.

\section{Discussion}

In our study group, the proportion of lung cancer in young female patients was higher than that in young male patients, with a male-to-female ratio of $1: 1.29$. This trend is mostly consistent with previous research results $(3,5)$. However, some studies found that the incidence of lung cancer in males is higher than that in females, with a male to female ratio of 1.03:1-2.4:1 $(11,15,16)$. This difference may be due to the younger age of young lung cancer patients included in our study. In their study, the upper age limit for patients included in the study was greater than 30 years, while the oldest age for patients in our study was 30 years. Besides, in the study by Liu et al, the ratio of male to female in the 20-30 age group was 1.03:1 (16). The lowest age of patients in their study was 20 years, while the lowest age of patients in our study was 11 years. May be the lower age limit is different, which also makes the ratio of male-to-female different. In addition, in previous studies, young patients were defined as 45 years old or under 40 years old. In our study, the patient age ranged from 11 to 30 years old, with an average age of 26 years. Liu et al. found that the average age was 28 years in the $20-30$ age subgroup (16), which was close to our results.

In this study, the rate of patients with a history of smoking was low (17.5\%), and only $5.6 \%$ were women. Previous studies have reported that smoking rates in young lung cancer patients range from $92 \%$ to $93 \%(17,18)$. However, with the establishment and enforcement of social smoking ban, the smoking rate of young people has also 


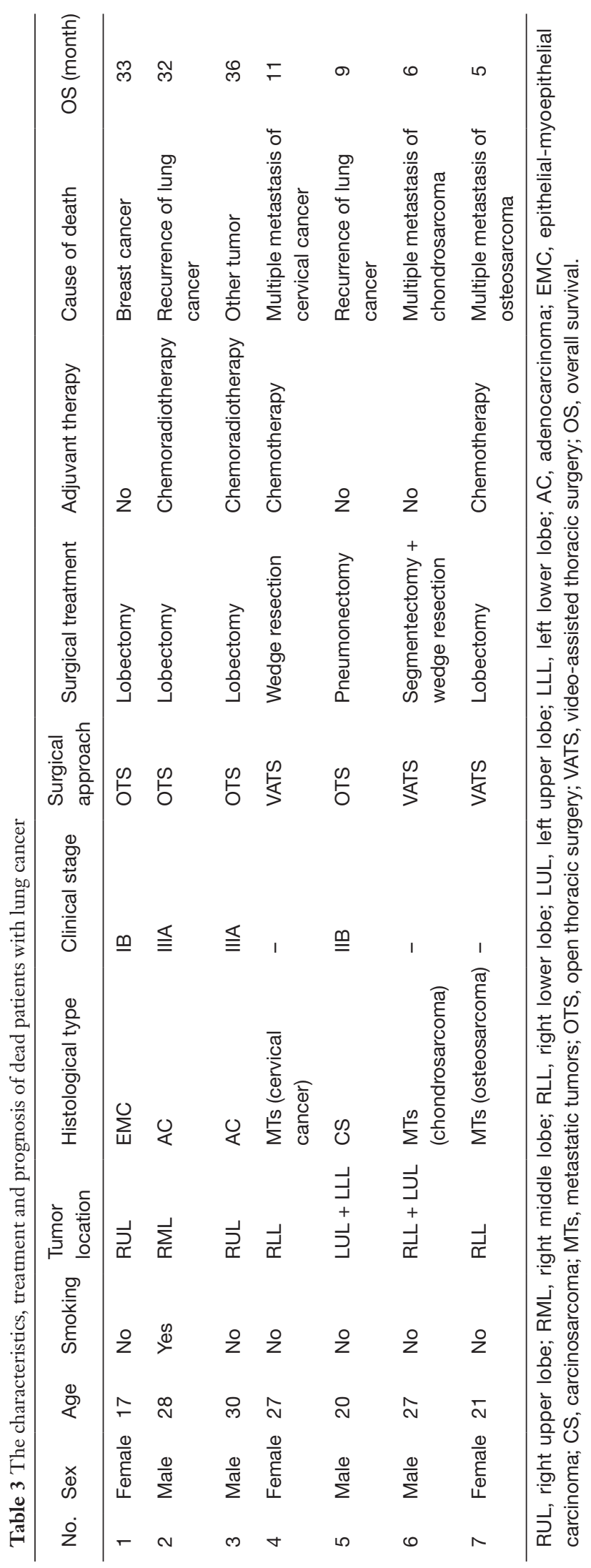

decreased (18). Our results also reflect the effectiveness of the comprehensive anti-smoking system.

In young patients, tumors were predominant on the right side compared with left side (53.4\% vs. $42.7 \%)$, which was consistent with other findings $(19,20)$. In our study, the most common tumors were located at the left lower lobe $(27.2 \%)$, followed by the right upper lobe $(21.4 \%)$ and the right lower lobe (21.4\%). In Freise's study, the most common tumors were located at the right upper lobe (19). This may be a feature of tumorigenesis in young lung cancer patients aged 30 and younger or it may be related to the limited sample size in the current group. In addition, the majority of patients in our study (58.3\%) were found to have small lung tumors and the maximum diameter of the tumors was $0-2 \mathrm{~cm}$. T stage was earlier and the relative prognosis was better, which is also a difference between young lung cancer patients and middle-aged and elderly patients.

Most studies have shown that adenocarcinoma is the most common histopathological type in young lung cancer patients $(2,3,21)$. Our study also found that nearly half of the patients had adenocarcinoma, which was consistent with the reported high incidence of lung adenocarcinoma. Surprisingly, although many previous studies reported rates of lung squamous cell carcinoma in young patients ranging from $3 \%$ to $35 \%(15,16,22,23)$, no patients with squamous cell carcinoma were found in our study. However, the patients included in our study were all younger than 30 years old, and only a few of them had a smoking history. Previous studies have shown that squamous cell carcinomas are more common in elderly patients, and most of these patients have smoking habits $(4,6,14)$. The absence of squamous cell carcinoma may also be due to the fact that all of our patients were surgically treated. The frequencies of small cell lung cancer and large cell lung cancer in our patient group were only $1.0 \%$. Yoneyama et al. found that $1.5 \%$ of young patients younger than 49 years old had small cell lung cancer (15). In the study by Xia et al., $2.7 \%$ of young lung cancer patients younger than 45 years old had small cell lung cancer, while in the control group, $4.3 \%$ of lung cancer patients older than 45 years old had small cell lung cancer, with no statistical difference between groups (24). Shimono et al. reported no type of small cell lung cancer in young lung cancer patients, which may be related to the relatively small number of cases in their study; only 24 young patients were younger than 39 years old (25). Although large cell lung cancer is an aggressive lung cancer subtype, its proportion in the lung cancer 
Table 4 Univariate and multivariate of OS in 95 cases

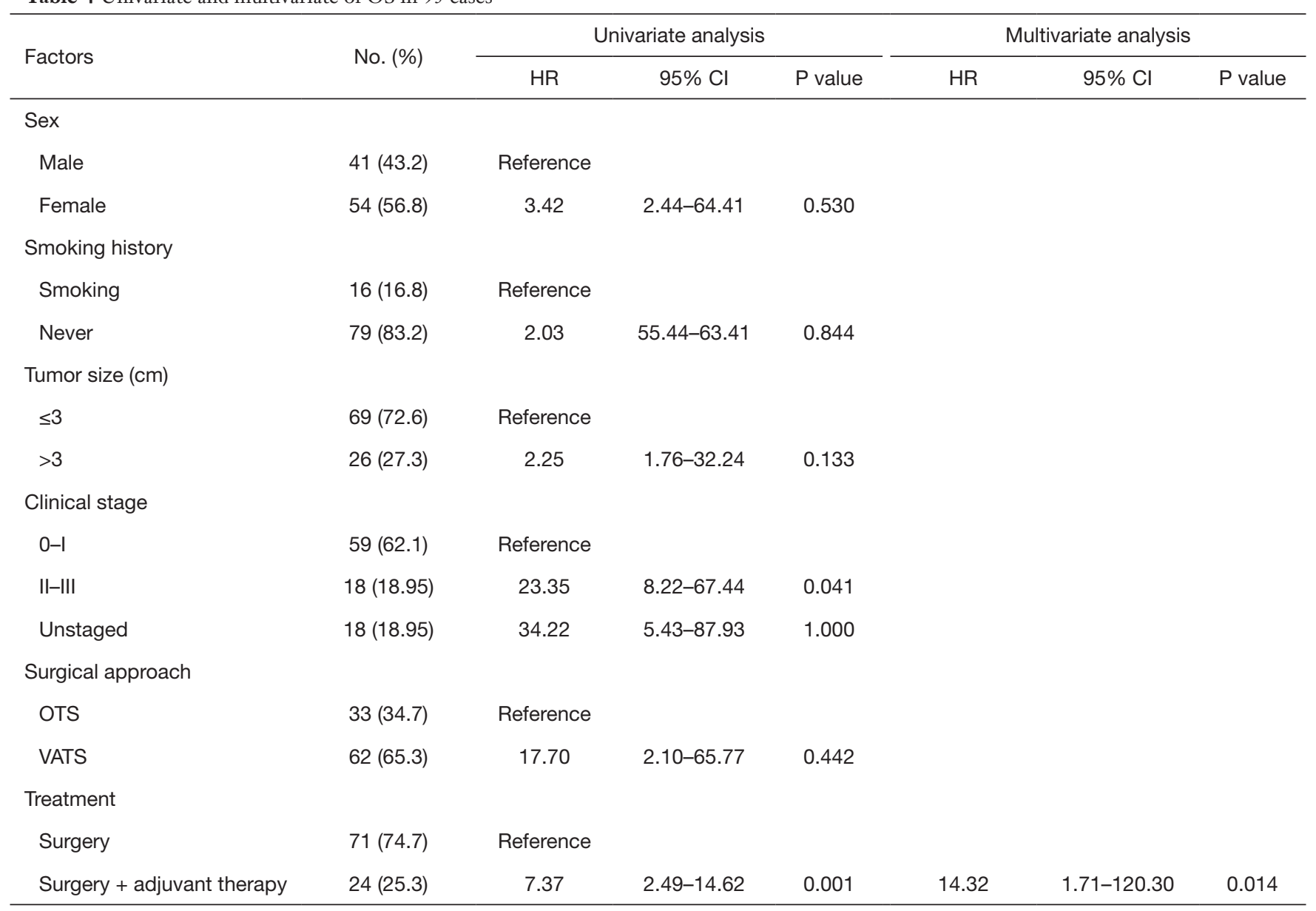

OS, overall survival; HR, hazard ratio; OTS, open thoracic surgery; VATS, video-assisted thoracic surgery.

population is relatively low. Fasano et al. reported that large cell lung cancer accounts for about 3.0\% of all lung cancer cases (26). In a large sample analysis of the population, Yang et al. reported that large cell lung cancer accounted for $0.9 \%$ of all non-small cell lung cancer patients from 2004 to 2009, while the ratio rose to $1.1 \%$ from 2010 to 2014 (27). In our study, mucoepidermoid and carcinoid tumors accounted for $10.7 \%$ and $7.8 \%$ of the patient group, respectively. Yoneyama et al. also found that carcinoids accounted for about $3.4 \%$ of lung cancer patients younger than 49 years old, but Bryant et al. reported that carcinoid accounted for $26 \%$ of lung cancer patients under 45 years old, while mucoepidermoid cancer accounted for $6 \%$ (23), which is not consistent with our results. This may be because the patients in our study are younger or may be due to racial differences, and more research is needed to explore the specific reasons.
Many previous studies have shown that lung cancer in young people is found late, progresses rapidly and has a poor prognosis $(10,15)$. However, some studies reported that the prognosis of young lung cancer patients after surgical resection is relatively good (28). In this study, only $15.5 \%$ of the patients were classified as stage IIB-IIIA, but no patients were identified at stage IV. Early studies by Bourke et al. found that most of the tumors in young lung cancer patients were diagnosed at advanced stage, with 77\% of patients in stage III-IV. In Kozielski's study, four out of six patients below 30 years old were stage III and showed a poor prognosis (4). Therefore, these results suggest that young people's low vigilance for lung cancer may lead to delays in cancer treatment. However, in our study, $47.6 \%$ of all patients were in clinical stage I, and $59.3 \%$ were in early stage 0-IIA. Most of the young lung cancer patients in our study were found through physical examination or 
screening, which suggests that early detection is important for young patients. The study from The National Lung Screening Trial Research Team suggests that low-dose computed tomography can achieve reduction in mortality from lung cancer (29). An Italian multicenter trial showed that long-term low-dose computed tomography screening programs could reduce lung cancer mortality by 39\% (30). In addition, young patients had a strong desire for treatment and actively accepted surgery as the main treatment. All of them had a good prognosis. In the follow-up period, except one young woman in stage IB died of breast cancer 33 months after operation, and all the other patients survived.

Current studies have shown that surgical treatment is still the preferred treatment for lung cancer. Because young lung cancer patients have good compensatory ability of organ function and strong ability to withstand surgical trauma, the performance of surgical treatment in this patient group is advantageous. In our study, except for one patient with pleural metastasis who only underwent biopsy, the other patients all received standard surgical treatment. Nearly half of the patients (47.6\%) underwent lobectomy with mediastinal lymph node dissection. Lung lobectomy with mediastinal lymph node dissection is recommended as a standard treatment for patients with early lung cancer. The 5-year survival rate of stage IA patients can reach $70 \%$ (31). In our study, $37.9 \%$ patients underwent sublobectomy (including anatomical segmentectomy and wedge resection). Although it is still controversial, sublobectomy has the same survival advantage as lobectomy in the treatment of early lung cancer, especially stage IA lung cancer, and more lung function is preserved for patients $(31,32)$.

In our study, $92.6 \%$ of patients survived and only $7.4 \%$ (7 patients) died in 95 patients who were followed up successfully. The 1-year and 3-year survival rates for the 95 cases were $95.6 \%$ and $85.9 \%$, respectively. Young lung cancer patients showed excellent prognosis, which is consistent with the results of Yoneyama et al. Their study found that the 5 -year survival rate of patients under 49 years of age was $81.5 \%$, the corresponding survival rates were $77.3 \%$ and $70.6 \%(\mathrm{P}=0.0001)$ in patients aged $50-69$ years and 70 or older, respectively (15). Of the seven patients in our study who died, only one died of recurrence of lung cancer, while the others died of recurrence of other tumors or primary tumors. Our study found that treatment modalities were significantly associated with the prognosis of young lung cancer patients $(\mathrm{P}<0.05)$, which was also consistent with the clinical stage of lung cancer. For early lung cancer, surgical treatment alone can achieve satisfactory prognosis. However, most of the patients who need adjuvant therapy such as chemoradiotherapy after operation are those with advanced stage. Whether they are treated with chemoradiotherapy or combined with molecular targeted therapy post-operation, the prognosis of advanced stage young lung cancer patients is still worse than that of patients with early lung cancer.

This study has several limitations, most notably its retrospective nature and single-institution design, which may lead to bias in patient selection. Another limitation is that the follow-up time of some patients was too short to fully analyze the long-term prognostic survival of patients, which requires a long-term follow-up in the later period to further analyze prognosis and its influencing factors. Besides, because of the limitations of retrospective study, there are some errors in the data collected on survival and prognosis, which may lead to statistical analysis deviation.

\section{Conclusions}

Our study shows that the main clinical pathological type of lung cancer in young patients aged 30 years and younger is adenocarcinoma, while low-grade malignant tumors such as mucoepidermoid carcinoma and carcinoid tumors also account for a certain proportion. Most of the clinical stages are in stage 0-IIA. Surgical treatment is the main method, while lobectomy is still the main method of surgery, and the prognosis is very good. With the popularization and importance of early diagnosis, the proportion of sublobectomy is increasing, and healthier lung tissues and functions are retained for patients. Because our research is a retrospective study and the sample size is limited, this study also has some limitations. More prospective largesample studies for younger lung cancer patients are required focusing on early diagnosis and treatment, post-relapse treatment and molecular, genetic and other basic aspects of research.

\section{Acknowledgments}

None.

\section{Footnote}

Conflicts of Interest: The authors have no conflicts of interest to declare. 
Ethical Statement: The authors are accountable for all aspects of the work in ensuring that questions related to the accuracy or integrity of any part of the work are appropriately investigated and resolved. This study was approved by the ethics committee of National Cancer Center/Cancer Hospital, Chinese Academy of Medical Sciences and Peking Union Medical College (approval number: 19/239-2023). The requirement of patients' informed consent was waived owing to the retrospective nature of the study.

\section{References}

1. Bray F, Ferlay J, Soerjomataram I, et al. Global Cancer Statistics 2018: GLOBOCAN Estimates of Incidence and Mortality Worldwide for 36 Cancers in 185 Countries. CA Cancer J Clin 2018;68:394-424.

2. Subramanian J, Morgensztern D, Goodgame B, et al. Distinctive characteristics of non-small cell lung cancer (NSCLC) in the young: a surveillance, epidemiology, and end results (SEER) analysis. J Thorac Oncol 2010;5:23-8.

3. Thomas A, Chen Y, Yu T, et al. Trends and characteristics of young non-Small cell lung cancer patients in the United States. Front Oncol 2015;5:113.

4. Kozielski J, Kaczmarczyk G, Porebska I, et al. Lung cancer in patients under the age of 40 years. Contemp Oncol (Pozn) 2012;16:413-5.

5. Arnold BN, Thomas DC, Kim AW, et al. Lung Cancer in the Very Young: Treatment and Survival in the National Cancer Data Base. J Thorac Oncol 2016;11:1121-31.

6. Corrales-Rodríguez L, Arrieta O, Mas L, et al. An international epidemiological analysis of young patients with non-small T cell lung cancer (AduJov-CLICaP). Lung Cancer 2017;113:30-36.

7. Pemberton JH, Nagorney DM, Gilmore JC, et al. Bronchogenic carcinoma in patients younger than 40 years. Ann Thorac Surg 1983;36:509-15.

8. Antkowiak JG, Regal A, Takita H. Bronchogenic carcinoma in patients under age 40. Ann Thorac Surg 1989;47:391-3.

9. Neuman HW, Ellis FH, McDonald JR. Bronchogenic carcinoma in persons under forty years of age. $\mathrm{N} \mathrm{Engl} \mathrm{J}$ Med 1956;254:502-7.

10. DeCaro L, Benfield JR. Lung cancer in young persons. J Thorac Cardiovasc Surg 1982;83:372-6.

11. Bourke W, Milstein D, Guira R, et al. Lung cancer in young adults. Chest 1992;102:1723-9.

12. Sugio K, Ishida T, Kaneko S, et al. Surgically resected lung cancer in young adults. Ann Thorac Surg 1992;53:127-31.

13. Roviaro GC, Varoli F, Zannini P, et al. Lung cancer in the young. Chest 1985;87:456-9.

14. Inoue $\mathrm{M}$, Okumura $\mathrm{M}$, Sawabata $\mathrm{N}$, et al. Clinicopathological characteristics and surgical results of lung cancer patients aged up to 50 years: the Japanese Lung Cancer Registry Study 2004. Lung Cancer 2014;83:246-51.

15. Yoneyama R, Saji H, Kato Y, et al. Clinicopathological characteristics and treatment strategies for young lung cancer patients. Ann Transl Med 2019;7:100.

16. Liu M, Cai X, Yu W, et al. Clinical significance of age at diagnosis among young non-small cell lung cancer patients under 40 years old: a population-based study. Oncotarget 2015;6:44963-70.

17. Ganz PA, Vernon SE, Preston D, et al. Lung cancer in younger patients. West J Med 1980;133:373-8.

18. Kozielski J, Kaczmarczyk G, Porębska I, et al. Lung cancer in patients under the age of 40 years. Wspolczesna Onkol 2012;16:413-5.

19. Freise G, Gabler A, Liebig S. Bronchial carcinoma and long-term survival. Retrospective study of 433 patients who underwent resection. Thorax 1978;33:228-34.

20. Ross P. Surgical therapy for lung cancer. Medical Update for Psychiatrists 1996;1:110-3.

21. Gitlitz B, Addario B, Sable-Hunt A, et al. PD1. 05 (also presented as P1. 49): the genomics of young emergent lung cancer. J Thorac Oncol 2016;11:S174.

22. Ramalingam S, Pawlish K, Gadgeel S, et al. Lung cancer in young patients: analysis of a surveillance, epidemiology, and end results database. J Clin Oncol 1998;16:651-7.

23. Bryant AS, Cerfolio RJ. Differences in outcomes between younger and older patients with Non-small Cell Lung Cancer. Ann Thorac Surg 2008;85:1735-9.

24. Xia J, Li H, Ji Y, et al. Clinicopathologic characteristics and EGFR mutations in lung cancer patients aged below 45 years. Curr Probl Cancer 2019;43:363-70.

25. Shimono T, Hayashi T, Kimura M, et al. Surgical treatment of primary lung cancer in patients less than 40 years of age. J Clin Oncol 1994;12:981-5.

26. Fasano M, Della CC, Papaccio F, et al. Pulmonary largecell neuroendocrine carcinoma: From epidemiology to therapy. J Thorac Oncol 2015;10:1133-41.

27. Yang Q, Xu Z, Chen X, et al. Clinicopathological characteristics and prognostic factors of pulmonary large cell neuroendocrine carcinoma: A large population-based analysis. Thoracic Cancer 2019;10:751-60.

28. Minami H, Yoshimura M, Matsuoka H, et al. Lung Cancer 
Treated Surgically in Patients $<50$ Years of Age. Chest 2001;120:32-6.

29. National Lung Screening Trial Research Team, Aberle DR, Adams AM, et al. Reduced lung-cancer mortality with low-dose computed tomographic screening. N Engl J Med 2011;365:395-409.

30. Pastorino U, Sverzellati N, Sestini S, et al. Ten-year results of the Multicentric Italian Lung Detection trial demonstrate the safety and efficacy of biennial lung cancer

Cite this article as: Li F, He H, Qiu B, Ji Y, Sun K, Xue Q, Guo W, Wang D, Zhao J, Mao Y, Mu J, Gao S. Clinicopathological characteristics and prognosis of lung cancer in young patients aged 30 years and younger. $\mathrm{J}$ Thorac Dis 2019;11(10):4282-4291. doi: 10.21037/jtd.2019.09.60 screening. Eur J Cancer 2019;118:142-8.

31. Wolf AS, Richards WG, Jaklitsch MT et al. Lobectomy versus sublobar resection for small (2 cm or less) non-small cell lung cancers. Ann Thorac Surg 2011;92:1819-23.

32. Whitson BA, Groth SS, Andrade RS, et al. Survival After Lobectomy Versus Segmentectomy for Stage I Non-Small Cell Lung Cancer: A Population-Based Analysis. Ann Thorac Surg 2011;92:1943-50. 